Diabetologia 10, $427-430(1974)$

(C) by Springer-Verlag 1974

\title{
Surface Electrostatic-Charge Measurements on Islet and Zymogen Granules : Effect of Calcium Ions
}

\author{
P.M. Dean* \\ Department of Pharmacology, University of Cambridge, Cambridge, England \\ Received: February 25, 1974, and in revised form: May 27, 1974
}

\begin{abstract}
Summary. Particle microelectrophoresis has ben used to measure the surface charge of secretory granules isolated from the exocrine and endocrine pancreas of guineapigs. Zymogen granules were found to have a surface charge density of 2378 esu.cm $^{-2}$ and islet granules 4177 esu.cm ${ }^{-2}$. Calcium ions decreased the strface charge density of both types of granule; binding of calcium at the
\end{abstract}

zymogen granule surface was found to fit a Langmuir adsorption isotherm. The results are discussed with respect to the role of calcium in exocytosis.

Key words: Particle microelectrophoresis, zymogen granules, islet granules, surface charge, zeta potential, calcium binding, exocytosis.
Electrostatic repulsive and Van der Waals attractive forces may be a major determinant in controlling adhesion of insulin secretory granules to the cell membrane prior to secretion by exocytosis $[8,9]$. In order to test this idea it is necessary to measure the electrostatic charge at the surface of the secretory granule. Particle microelectrophoresis is a simple quantitative method which can readily be applied to this problem and has already been employed successfully to investigate the ionogenic nature of the chromaffin granule surface [11]. Calcium ions are of critical importance in the mechanism of exocytosis and in this paper the effects of these divalent ions on surface charge have been studied using secretory granules isolated from the islets of Langerhans and zymogen granules from the acinar tissue of guinea-pig pancreas.

\section{Methods}

\section{Isolation of Secretory Granules}

a) Islet Granules. In each experiment the pancreata from two guinea-pigs ( $800 \mathrm{~g}$ weight) were used; the animals had previously been fed ad libitum. Islets of Langerhans were isolated by collagenase digestion of the surrounding acinar tissue, using the method of Lacy and Kostianovsky [1]. Approximately 150 islets were isolated from each pancreas. The isolated. islets were homogenized in $0.4 \mathrm{ml}$ of sucrose solution $(0.3 \mathrm{M})$ at $4^{\circ} \mathrm{C}$ and buffered with sodium phosphate $(5 \mathrm{mM})$ at pH 6.0; secretory granules were harvested by the centrifugation procedure of Howell et al. [5]. The pellet of islet secretory particles was resuspended by homogenization in $0.8 \mathrm{ml}$ sucrose $(0.3 \mathrm{M})$ at $\mathrm{pH} 6.0$ and maintained at $4^{\circ} \mathrm{C}$. No further subdivision of the islet granule fraction was attempted.

b) Zymogen Granules. Two guinea-pigs of either sex and weighing $800 \mathrm{~g}$ were used for each preparation.

\footnotetext{
* Beit Memorial Research Fellow.
}

The pancreata were removed and the zymogen granules isolated from them by the method of Jamieson and Palade [6].

The purity of the secretory granule pellet for both the islet and acinar granule fractions was checked by electron microscopy.

\section{Electrophoretic Mobility Determinations}

The electrokinetic properties of the secretory granules were studied with a micro-electrophoresis apparatus; the method has been described previously [11]. Mobility determinations were made in $0.16 \mathrm{M} \mathrm{KCl}$ and the $\mathrm{pH}$ of the solution adjusted to 6.0 for islet granules, or 7.0 for zymogen granules, by adding appropriate amounts of $0.16 \mathrm{M} \mathrm{KOH}$ or $0.16 \mathrm{M} \mathrm{HCl}$. Mean mobilities of the secretory granules, using forward and reverse polarity, were determined at $25^{\circ} \mathrm{C}$ from 10 readings on 4 separate granule preparations; mobility values are expressed in $\mu \mathrm{m} . \mathrm{s}^{-1} . \mathrm{cm} \cdot \mathrm{v}^{-1}$. In studying the effect of $\mathrm{Ca}^{2+}$ ions on mobility, aliquots of a $\mathrm{Ca} \mathrm{Cl}_{2}$ solution (1M) were added to a solution of KCl to maintain a constant ionic strength of 0.16 eq.lit ${ }^{-1}$.

\section{Theory}

A charged particle placed in an ionic solution adsorbs a layer of ions on to its surface, forming a relatively fixed layer; ions further away from the surface are mobile. The electrical potential in the slipping plane between the fixed and flowing liquid is determinative for electrokinetic phenomena. This potential is called the zeta potential and is related to the mobility of the particle in an electric field by the equation:

$$
\zeta=\frac{4 \pi \eta \mu}{\varepsilon}
$$

where $\zeta$ is the zeta potential in volts, $\eta$ is the viscosity of the solution in Poise, $\mu$ is the electrophoretic mobi- 
lity $\mathrm{cm} \mathrm{sec} \mathrm{e}^{-1} \cdot \mathrm{V}^{-1} \mathrm{~cm}, \varepsilon$ is the dielectric constant of the solution. When $\zeta$ is $<25 \mathrm{mV}$ the measured zeta potential approximates to the surface potential. The surface charge density of the particle, $\sigma$ in esu.cm-2 is related to the mobility by the equation: -

$$
\sigma=\left\{\frac{N \varepsilon k T}{2000 \pi} \sum_{i} C_{i}\left[\exp \left(-\frac{1200 \pi e \eta z_{i} \mu}{\varepsilon k T}\right)-1\right]\right\}^{1 / 2}
$$

where $\mathrm{N}$ is the Avogadro number, $k$ is Boltzman's constant erg $/ K^{0}, T$ is the temperature $K^{0}, C_{i}$ and $Z_{i}$ are respectively the molar concentration and the valency of ions of the $i$ th species and $e$ is the electron charge in esu,

Usings Stern's model [13] for the adsorption of ions on to charged surfaces, an expression for the decrease in surface charge density caused by adsorption of counter-ions can be obtained:

$$
\Delta \sigma_{\mathrm{Ca}^{2+}}=2 e n_{\mathrm{Ca}^{2+}}=\frac{2 e N_{\mathrm{a}}}{1+\exp \left(-\Delta G_{\mathrm{Ca} /} / k T\right) / \chi_{\mathrm{Ca}}}
$$

where $\Delta_{\mathrm{Ca}^{2+}}$ is the decrease in surface charge density, $n_{\mathrm{Ca}^{2+}}$ is the number of adsorbed $\mathrm{Ca}^{2+}$ ions $\mathrm{cm}^{-2}$, $N_{\mathrm{a}}$ is the number of available adsorption sites, $\Delta G_{\mathrm{Ca}^{2+}}{ }^{2+}$ is the electrochemical free energy of adsorption of $\mathrm{Ca}^{2+}$, and $\chi_{\mathrm{Ca}}$ is the molar fraction of $\mathrm{Ca}^{2+}$, equal to concentration of $\mathrm{Ca}^{2+}$ ion/55.6, where 55.6 is the mole fraction water. Substituting $K$ for the expression:

exp. $\frac{\left(\Delta G_{\mathrm{Ca}^{2+}} / k T\right)}{55.6}$ in equation (3) and rearranging yields equation (4), the linear form of the Langmuir adsorption isotherm

$$
\frac{1}{\Delta \sigma_{\mathrm{Ca}^{2+}}}=\frac{1}{2 e N_{\mathrm{a}}}+\frac{1}{C_{\mathrm{Ca}^{2+}}} \cdot \frac{1}{2 e N_{\mathrm{a}} K}
$$

Hence, if a plot of $1 / \Delta_{\mathrm{Ca}^{2+}}$ against a $1 / C_{\mathrm{Ca}^{2+}}$ yields a straight line, the number of available calcium ion binding sites on the granule surface can be obtained (3). The same method has also been applied by Seaman, Vassar and Kendal [12] to the erytrocyte surface.

\section{Results}

Secretory granules from the islets of Langerhans had an electrophoretic mobility of $-1.17 \pm \operatorname{SEM} 0.06(n=$ 4) compared with a lower value of $-0.67 \pm$ SEM 0.04 $(n=6)$ for the zymogen granules. Proportionate differences were found for the zeta potential, i.e., -15.0 $\pm 0.8 \mathrm{mV},-8.6 \pm 0.6 \mathrm{mV}$ and surface charge density i.e., $4177 \pm 214$ esu.cm-2, $2378 \pm 154$ esu.cm $^{-2}$ respectively for islet and zymogen granules.

Progressively increasing the $\mathrm{Ca}^{2+}$ concentration of the suspending $\mathrm{KCl}$ solution decreased the electrophoretic mobility of both types of secretory granule. The relationship between surface charge density and calcium concentration is shown in Figure 1. A plot of the reciprocal of the decrease in surface charge density against $1 / \mathrm{Ca}^{2+}$ concentration produced a straight line for zymogen granules, with a slope of $0.33 \times 10^{-5}$ esu.-1 $\mathrm{cm}^{-2}$ moles. 1.-1 $^{-1}$ and an intercept of $0.41 \times 10^{-3} \mathrm{esu}^{-1}$ $\mathrm{cm}^{2}$ (Fig. 2). The number of available calcium binding sites was calculated from equation 4 to be $2.51 \times 10^{12}$ $\mathrm{cm}^{-2}$ and the electrochemical free energy of calcium ion adsorption, $\Delta G_{\mathrm{Ca}}$, was $-5.23 \mathrm{KCals}$. mole-1. A similar double reciprocal plot for calcium binding to islet granules did not yield a straight line and analogous calculations could not be made.

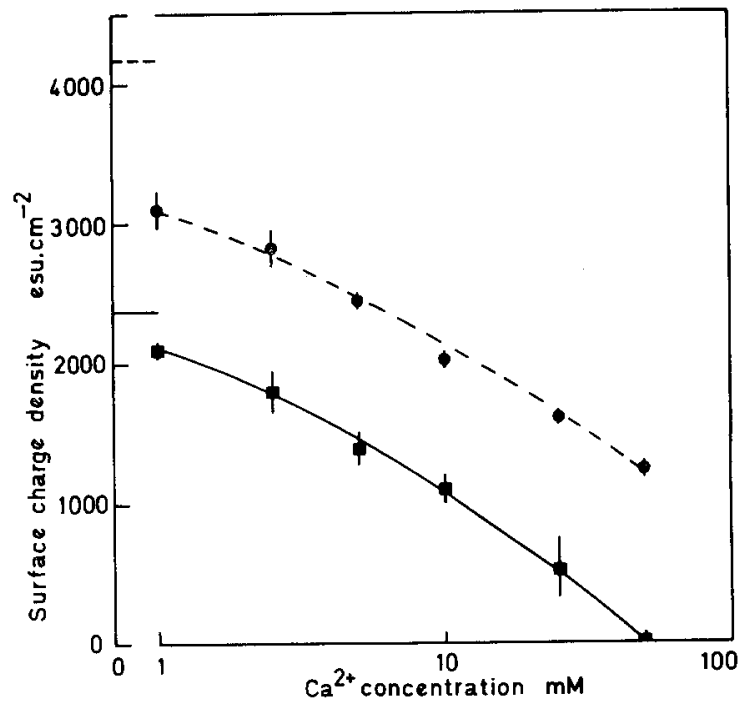

Fig. 1. The effect of calcium-ion concentration on electrostatic surface charge density of islet granules (broken line) and zymogen granules (full line). The surface charge density of the granules in $0.16 \mathrm{M} \mathrm{KCl}$ in the absence of calcium is shown to the left of the curves. SEM's $(n=40)$ shown by the vertical bar

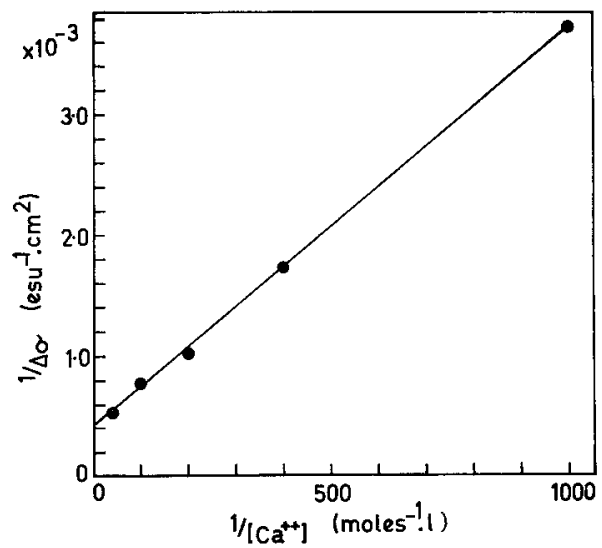

Fig. 2. Langmuir adsorption isotherm for the binding of calcium ions to zymogen granules. Double reciprocal plot of decrease in surface charge density against calcium concentration

\section{Discussion}

A surface negative charge appears to emerge as characteristic of isolated secretory granules; islet granules, zymogen granules, chromaffin granules [11, 3 ), and neurohypophyseal granules (unpublished data), 
all have negative zeta potentials between $-8 \mathrm{mV}$ and $-16 \mathrm{mV}$. The contribution of surface cationic groups to the total charge has yet to be determined, although like red cells [4] the chromaffin granule surface behaves essentially as a polyanion. Structural instability of islet and zymogen granules at low $\mathrm{pH}$ precluded measurements of surface $\mathrm{pK}$ values; chromaffin granules, on the other hand, have a $\mathrm{pK}_{\mathrm{a}}$ which is consistent with ionization of surface carboxyl groups.

The islets of Langerhans contain two major types of secretory granule, $\beta$-granules containing stored insulin and $\alpha$-granules containing glucagon. No attempt has been made in this study either to separate the two fractions or to assess the relative contribution of each type to the measurement of mean granule mobility. The standard deviation for 40 determinations of mobility was small, $15 \%$, and of a single distribution, indicating either that one type of granule is predominant, or that the surface charge of $\alpha$-and $\beta$-granules is equivalent. Any attempt to consider this data as pertaining to $\beta$-granules alone must be viewed with caution. Different calcium binding properties of the two granule types may have been the cause of the non-linearity of the Langmuir adsorption isotherm; a similar plot for the monodispersed preparation of zymogen granules produced the expected straight line.

Nevertheless, if, as seems likely, one can assume that the surface potential arises from discrete electron charges $[11,3]$, it is possible (knowing the granule size) to estimate the net number of surface ionic groups. For guinea-pig zymogen granules of diameter $391 \mathrm{~nm}$ (Dean, unpublished data) there would be 23,777 electronegative sites per granule, with a mean area per site of $20 \mathrm{~nm}^{2}$, and a mean anionic site separation of $4.8 \mathrm{~nm}$. In these terms the number of $\mathrm{Ca}^{2+}$ binding sites on the zymogen granule surface is 12,100 . Correspondingly, islet granules, of diameter $290 \mathrm{~nm}$ [1], have 22,980 excess charges, a mean site area of $11.5 \mathrm{~nm}^{2}$ and intersite separation distance of $3.6 \mathrm{~nm}$. These values assume importance in any consideration of the mechanism of binding of secretory granules to the cell membrane, e.g. calcium cross-linkage, in the adhesion stage of exocytosis [8]. For example, in the simplest case, consider the zymogen granule to be a rigid sphere binding to a planar surface by calcium bridges: the number of anionic sites present on a polar cap $0.2 \mathrm{~nm}$ thick (the calcium ionic diameter) can be calculated to be 12 , and an upper limit of 6 specific calcium-ion binding sites may reasonably be expected to be available for adhesive cross-linking. By the same reasoning the number of electronegative sites presented by the pole of an islet granule to a planar surface would be $a$ maximum of 16. Such calculations necessarily assume an even surface distribution of ionogenic sites and would not apply if significant clustering could be demonstrated.

Islet granules were more sensitive to $\mathrm{Ca}^{2+}$ ions than the zymogen granules; at $1 \mathrm{mM} \mathrm{Ca}{ }^{2+}$ the surface charge was reduced by $25 \%$ and $11 \%$ respectively. It has pre- viously been shown from electrophysiological studies of islet cells that substances which induce insulin secretion evoke an electrically gated $\mathrm{Ca}^{2+}$ influx across the cell membrane [2]. The quantity of $\mathrm{Ca}^{2+}$ entering a cell during the action potential can be estimated to be $0.1 \mathrm{pmole} / \mathrm{cm}^{2}[10]$, and one action potential would increase the total cellular $\mathrm{Ca}^{2+}$ by $0.68 \mu \mathrm{M}$. However the concentration transient close to the membrane inner surface during the time course of the action potential is likely to be considerably larger than $0.68 \mu \mathrm{M}$. The finding that $\mathrm{Ca}^{2+}$ binds to secretory granule surfaces in solutions of total ionic strength comparable with that expected intracellularly poses the important question of the role of $\mathrm{Ca}^{2+}$ in the excitation-secretion coupling mechanism of exocytosis. Matthews [8, 9] first proposed the hypothesis that if in the islet cells the secretory granules and the cell membrane inner surface are equally charged, then an electrostatic repulsive force-field would be generated when a secretory particle approached the membrane. Unless the kinetic energy of translational motion exceeds the electrostatic energetic barrier, contact-collision of the granule would not occur. It was postulated that one function of $\mathrm{Ca}^{2+}$ ions in exocytosis is to neutralise partially the surface negative charges and consequentially diminish the energetic barrier only those grauules with sufficiently large kinetic energies would make lasting contact with the membrane thus permitting adhesion. In order to test this hypothesis, the attractive force constant between granules and the cell membrane has to be ascertained before it is possible to build a kinetic model for secretion by exocytosis based on the energetic considerations of granule-membrane interaction.

Acknowledgement. I thank Keith Matthews for continued encouragement in this work.

\section{References}

1. Dean, P.M.: UItrastructural morphometry of the pancreatic $\beta$-cell. Diabetologia 9, 115-119 (1973)

2. Dean, P.M., Matthews, E.K.: Glucose-induced electrical activity in pancreatic islet cells. J. Physiol. 210, $255-264(1970)$

3. Dean, P.M., Matthews, E. K. : Calcium-ion binding to the chromaffin granule surface. Biochem. J. (In Press)

4. Heard, D.H., Seaman, G.V.F.: The influence of $\mathrm{pH}$ and ionic strength on the electrokinetic stability of the human erythrocyte membrane. J. gen. Physiol. 43, 635-654 (1960)

5. Howell, S.L., Fink, C.J., Lacy, P.E.: Isolation and properties of secretory granules from rat islets of Langerhans. 1. Isolation of a secretory granule fraction. J. Cell. Biol. 41, 154-161 (1969)

6. Jamieson, J.D., Palade, G.E.: Intracellular transport of secretory proteins in the pancreatic exocrine cell. J. Cell. Biol. 34, 577-596 (1967)

7. Lacy, P.E., Kostianovsky, M.: Method for the isolation of intact islets of Langerhans irom the rat pancreas. Diabetes 16, 35-39 (1967)

8. Matthews, E. K. : Calcium and hormone released. In: Calcium and Cell Function, ed. Cuthbert, A. W. p. 163. London: Macmillan. 1970 
9. Matthews, E.K.: Electrical activity in islet cells and insulin secretion. Acta Diabet. Lat. 7, 83-89 (1970)

10. Matthews, E.K., Dean, P.M., Sakamoto, Y.: The bioelectrical activity of the islet cell membrane. In. Handbook of Experimental Pharmacology. Vol. 32/2. Insulin 2. (Ed Hasselblatt, A. \& Von Bruckhausen.) Berlin, Heidelberg, New York: Springer-Verlag (In Press)

11. Matthews, E.K., Evans, R.J., Dean, P.M.: The ionogenic nature of the secretory granule surface. Electrokinetic properties of isolated chromaffin granules. Biochem. J. 130, 825-832 (1972)
12. Seaman, G.V.F., Vassar, P.S., Kendall, M.J.: Calcium-ion binding to blood cell surfaces. Experientia $25,1259-1260(1969)$

13. Stern, O., Zur Theorie der Elektrolytischen Doppelschicht. Z. Elektrochem. 30, 508-516 (1924)

Dr. P.M. Dean,

Dept. of Pharmacology

Univ. of Cambridge

Medical School

Hills Road

Cambridge CB2 2QD

England 